\title{
Secretory products of macrophages: twenty-five years on
}

\author{
Carl Nathan \\ Department of Microbiology and Immunology, Weill Cornell Medical College, New York, New York, USA.
}

\begin{abstract}
No longer do scientists look down on macrophages as "garbage men" that act "nonspecifically." Last fall's Nobel Prizes honored two of the few scientists who studied macrophages three decades ago. Now perhaps thousands do, and the subtypes they describe reflect ongoing discoveries of macrophages' extraordinary plasticity.
\end{abstract}

\section{Why look back?}

When the editors of JCI asked me to comment on the impact of a Perspective I wrote 25 years ago (1), I had three reactions. Rereading the piece, I was flooded with memories of what it was like to survey the literature in an era before Google, PubMed, and EndNote: an aching back from carrying stacks of journals to the photocopy machine; the monotonous drone and scent of ozone as the bulb swept beneath the glass on which I forced down the volumes, hoping the text would be legible where it curved toward the binding. Then I wondered how researchers with the tools of the time managed to generate the mountain of knowledge that the Perspective surveyed. Around then, for example, my colleagues and I determined the sequence of a cDNA encoding inducible nitric oxide synthase (2) by electrophoresing countless Sanger reactions and laying a ruler across the gels. One of us called off As, Ts, Cs, and Gs until the relative positions of the furthest-migrating bands grew indistinct. Another typed the letters into a device introduced in the lab just a few years before, a personal computer.

My third reaction to the invitation, and the reason to accept it, was surprise that so many people had found cause over the years to read the Perspective. Why did it strike a chord? This is the point of the present Hindsight - not nostalgia for teamwork in a time when everything seemed harder, but recognition that there are times when our line of sight pivots: we are still looking ahead, but face different vistas. The act of taking stock can mark and perhaps contrib-

Conflict of interest: The author has declared that no conflict of interest exists.

Citation for this article: J Clin Invest. 2012; 122(4):1189-1190. doi:10.1172/JCI62930. ute to redirection. Here are some interrelated ways in which the field began to turn.

\section{Jettisoning junk}

At a time when textbooks called macrophages "the garbage men of the body," you could expect most immunologists and cell biologists to turn up their noses and study something else. Similarly, until recently, some called noncoding regions of the genome “junk DNA.” In 1987 it began to be clear that there is no hierarchy among biological phenomena in terms of their potential to yield important insights; there are only degrees of our ignorance. Little in nature is junk, even that which needs to be degraded. The processes by which tissues, cells, organelles, and molecules qualify for degradation and are recognized and disposed of are now among the most exciting frontiers in biology and therapeutics. There is much to learn from how macrophages do these things. The 1987 Perspective stressed how much more these cells do besides, for example, tissue trophism and host missed as garbage men.

\section{Nullifying non-specificity}

Before 1987, most immunologists coupled the "garbage men" sobriquet with an even more disparaging view, that macrophages, and along with them all the cells that constitute what is now called the innate immune system, were nonspecific. This constraint on vision was self imposed, under the assumption that a successful paradigm devalued phenomena that did not conform. In 2011, Bruce Beutler was honored with a Nobel Prize in Medicine or Physiology for demonstrating that macrophages use specific molecules to recognize other specific molecules generated by or in response to microbial pathogens. Jules defense. No more are macrophages dis-
Hoffmann shared the award for demonstrating the same principle in flies. The specificity of these interactions was simply somewhat broader than that to which students of T and B cells were accustomed.

The misconception of non-specificity in innate immunity began to take another hit when the 1987 Perspective called attention to the diverse signaling functions of reactive oxygen intermediates (ROI) (3), for which no coherent explanation existed. The challenge grew more acute with the explosion of knowledge over the next five years that introduced to medical biology a parallel set of reactive nitrogen intermediates (RNI), along with evidence that ROI and RNI produced by activated macrophages could both signal and kill (4). That apparent paradox was held temporarily at bay by the idea that low-output production of ROI and RNI was involved in signaling, while highoutput production was catalyzed by different enzymes and led to killing (4). However, assigning production to different enzymes did not resolve how signaling could occur without specificity. Moreover, by the early 2000s, my colleagues and I found that the same isoforms of ROI- and RNI-producing enzymes could both signal and kill (5).

In 2003 another Perspective I contributed to the JCI offered a resolution to these apparent paradoxes by introducing the concept of distinct kinds of specificity (5). "Molecular specificity" characterizes the interactions of molecular receptors with their specific molecular ligands, as in the work of Hoffmann and Beutler. Whether the ligands for a given receptor are single or multiple, interactions are restricted to a small set and propagate down largely private pathways by a succession of intermolecular handshakes. By contrast, ROI and RNI signal with atomic specificity. They react only with a few types of atoms and just with some of the atoms of each type. For example, among the most frequent targets of ROI and RNI in proteins are sulfur atoms in sulfhydryl side chains of cysteine residues with low $\mathrm{pKa}$. These are mostly active-site cysteines, such as in protein 
phosphatases, whose transient inhibition by ROI or RNI augments agonist-stimulated protein phosphorylation. The few atomic targets are widely represented in diverse macromolecules. This enables ROI and RNI to serve as diffusible regulators whose levels report the cell's metabolic state and whose covalent, usually reversible reactions help integrate diverse signaling paths with the cell's metabolic budget (5).

\section{Growing granularity}

The remarkable repertoire of macrophage secretory products and functions (1) presaged an explosion in the characterization of macrophage subsets. The most striking example was the recognition of a differentiation state of mononuclear phagocytes that conferred such distinctive properties that a new cell type was proposed - dendritic cells (6). The later finding that some dendritic cells arise from non-myeloid precursors reinforced the view that a novel cell type had been found. For this discovery, Ralph Steinman shared the 2011 Nobel Prize with Hoffman and Beutler. In the same vein, the pre-1987 subsets of "resident," "activated" (7), and "deactivated" (8) macrophages were subsequently joined with, renamed as, and/ or subdivided into "classically activated" and "alternatively activated" macrophages; "M1" or "M2" macrophages; and tumorassociated macrophages of multiple varieties - "invasive," "immunosuppressive," "activated," "perivascular," "angiogenic," and "metastatic" (9) - to name a few. The belief that tissue macrophages are irrevocably and terminally differentiated gave way to recognition of their replicative potential and functional plasticity.

\section{Aspiring to integration}

Perhaps the most important impetus of the 1987 Perspective was encouragement to integrate a wide spectrum of information from outside one's own focus. Today the goal is not just to integrate as wide a literature as one can manage to read (e.g., refs. 1 , $3-5,10,11)$ but to harness vastly increased computational power to integrate large numbers and diverse types of primary observations (12). Equally challenging, medicine needs to integrate submolecular structure (including, among other things, the sequence of DNA), molecular function, subcellular organization, cellular performance, organ physiology, and the behavior of individuals and populations.

On such a difficult journey it may be useful to equip ourselves with hindsight. In biology, there are things that need to go, but little "junk.” An organism's chemical reactions and interactions all represent information. All information is specific, but there are different kinds of specificity. Cells adapt better when they integrate as much as possible of the information available to them. Scientists do, too.

\section{Acknowledgments}

Many thanks go to Aihao Ding for careful reading of a draft of this article. The
Department of Microbiology and Immunology is supported by the William Randolph Hearst Foundation.

Address correspondence to: Carl Nathan, Weill Medical College, Box 62, 1300 York Avenue, New York, New York 10065, USA. Phone: 212.746.6505; Fax: 212.746.8587; E-mail: cnathan@med.cornell.edu.

1. Nathan CF. Secretory products of macrophages. J Clin Invest. 1987;79(2):319-326.

2. Xie QW, et al. Cloning and characterization of inducible nitric oxide synthase from mouse macrophages. Science. 1992;256(5054):225-228.

3. Nathan C. Epidemic inflammation: pondering obesity. Mol Med. 2008;14(7-8):485-492.

4. Nathan C. Nitric oxide as a secretory product of mammalian cells. FASEB J. 1992;6(12):3051-3064.

5. Nathan C. Specificity of a third kind: reactive oxygen and nitrogen intermediates in cell signaling. J Clin Invest. 2003;111(6):769-778.

6. Steinman RM, Cohn ZA. Identification of a novel cell type in peripheral lymphoid organs of mice. I. Morphology, quantitation, tissue distribution. J Exp Med. 1973;137(5):1142-1162.

7. Nathan CF, Murray HW, Wiebe ME, Rubin BY. Identification of interferon-gamma as the lymphokine that activates human macrophage oxidative metabolism and antimicrobial activity. J Exp Med. 1983;158(3):670-689.

8. Tsunawaki S, Sporn M, Ding A, Nathan C. Deactivation of macrophages by transforming growth factor-beta. Nature. 1988;334(6179):260-262.

9. Qian BZ, Pollard JW. Macrophage diversity enhances tumor progression and metastasis. Cell. 2010;141(1):39-51.

10. Nathan C. Aligning pharmaceutical innovation with medical need. Nat Med. 2007;13(3):304-308.

11. Nathan C, Ding A. Nonresolving inflammation. Cell. 2010;140(6):871-882.

12. Schadt EE, Bjorkegren JL. NEW: network-enabled wisdom in biology, medicine, and health care. Sci Transl Med. 2012;4(115):115rv1. 